\title{
ESTUDIO DE LA DIVERSIDAD GENÉTICA DE 20 ACCESIONES DE CACAO (Theobroma cacao L.) MEDIANTE AP-PCR DE LA COLECCIÓN DEL CENTRO DEL CACAO DE AROMA TENGUEL EN LA FINCA EXPERIMENTAL LA BUSETA
}

\author{
Mercedes Susana Carranza Patiño ${ }^{1}$, Emerick Motte $^{2}$, Virna Cedeño ${ }^{2}$, Olry Fernando Cevallos Falquez ${ }^{1}$, \\ Silvia Gicela Saucedo Aguiar ${ }^{1}$ y Hayron Fabricio Canchignia Martínez ${ }^{1}$ \\ ${ }^{1}$ Unidad de Investigación Cientifica y Tecnológica, Universidad Técnica Estatal de Quevedo. Av. Walter Andrade. \\ Km 11/2 vía a Santo Domingo, C.P. 73. Quevedo,Los Ríos, Ecuador. susycespinosa@yahoo.es \\ ${ }^{2}$ Laboratorio del Programa de Biotecnología, Universidad de Guayaquil. Guayaquil, Guayas, Ecuador.
}

\section{RESUMEN}

El presente estudio consistió en determinar la diversidad genética basada en los marcadores RAPD's (Random Amplification of Polymorphic DNA) de 20 accesiones de cacao (Theobroma cacao L.) de la variedad Nacional, con características de productividad, y niveles de resistencia, susceptibilidad y tolerancia a las principales enfermedades causadas por hongos como Ceratocistys fimbriata, Moniliophtora roreri y Crinipelis perniciosa. Este germoplasma de cacao se encuentra localizado en el Centro de Cacao de Aroma Tenguel, en la Finca Experimental "La Buseta" propiedad de la Universidad Técnica Estatal de Quevedo. La extracción de ADN se la realizó utilizando el protocolo de Doyle \& Doyle (1990) con algunas modificaciones. 14 oligonucleótidos fueron utilizados para la obtención de marcadores RAPD's, de los cuales 9 amplificaron productos reproducibles OPA-15, OPC-07, OPC-9, OPC-4, OPC-3, OPC-1, OPA-12 OPC-13 y OPA-7. Los productos de amplificación fueron migrados en geles de agarosa al $1.2 \%$ a 90 voltios por una hora. Los marcadores moleculares fueron analizados por medio de una matriz de datos binarios para calcular las distancias genéticas. Los nueve cebadores utilizados generaron 67 bandas de las cuales 59 (88\%) fueron polimórficas. El dendrograma mostró dos grupos A y B, en el grupo A se incluyo dos accesiones, y en el grupo B se encuentran los 18 restantes el cual incluye 2 subgrupos B1 y B2 en el subgrupo B1 se incluye la accesión (L-22-H-40), y en el subgrupo B2 están incluidas las accesiones que poseen características de productividad. El nivel de diversidad más alto se obtuvo con los oligonucleótidos OPC 04 (0.80), OPC 07 (0.82). El cebador OPC 01 (0.37) reportó el nivel más bajo. Las accesiones (L-22-H-40) (O.75), (L-34-H-07) (0.86) y el (L-42-H-60) (0.72) presentaron los niveles de variabilidad más altos, siendo los valores más bajos los mostrados por las accesiones (L-26-H-64) (O.48) y (L-23-H63) (O.47). El valor de la diversidad total (HT) obtenida para las 20 accesiones de T. cacao mediante RAPD fue de 0.636 .

Palabras claves: RAPD, ADN, cebadores y dendrograma, diversidad genética, polimorfismo.

\begin{abstract}
The present study consisted of determining the genetic diversity based on the scoreboards RAPD's (Random Amplification of Polymorphic DNA) of 20 accessions of cocoa (Theobroma cocoa L.) of the National variety, with characteristics of productivity, and levels of resistance, susceptibility and tolerance to the principal diseases caused by fungi as Ceratocistys fimbriata, Moniliophtora roreri and Crinipelis perniciosa. This germoplasma of thief is located in the Center of Cocoa of Aroma Tenguel, in the experimental estate "The Buseta" property of the Technical State University of Quevedo. The extraction of DNA realized it using the protocol of Doyle and Doyle (1990) with some modifications. 14 oligonucleótidos were used for the obtaining scoreboard RAPD's, of which 9 amplified reproducible products OPA-15, OPC-07, OPC-9, OPC-4, OPC-3, OPC-1, OPA-12 OPC- 13 y OPA-7. The products of amplification were migrated in gels of agarosa to $1.2 \%$ to 90 volts for an hour. The molecular scoreboards were analyzed by means of a counterfoil of binary information to calculate the genetic distances. Nine used powderhorns generated 67 bands of which 59 (88 \%) was polymorphic. The dendrograma showed two groups A and B, in the group A include two accessions, and in the group B the 18 remaining ones are which includes 2 subgroups B1 and B2 in the subgroup B1 includes the accession (L-22-H-40), and in the subgroup B2 there are included the accessions that possess characteristics of productivity. The level of diversity more high were obtained with the oligonucleótidos OPC 04 (0.80), OPC 07 (0.82). The powder-horn that brought (reported) the lowest level was OPC 01 (0.37). The accessions that presented the levels of variability more high places were the (L-22-H-40) (Or 75), (L-34-H-07) (0.86) and the (L-42-H-60) (0.72) whereas the lowest values them showed the accessions (L-26H-64) (Or 48) and (L-23-H63) (Or 47). The value of the total diversity (HT) obtained for 20 accessions of T. cocoa by means of RAPD was of 0.636 .
\end{abstract}

Key words: RAPD, DNA, primer and dendrograma, genetic diversity, polymorphism.

Recibido: Abril, 2007. Aceptado: Noviembre: 2007.

Publicado como ARTÍCULO en Ciencia y Tecnología 1: 1-5. 2008. 


\section{INTRODUCCIÓN}

El cacao (Theobroma cacao L.) es una de las principales plantas perennes de uso agroindustrial que se cultiva en la mayoría de países que se encuentran en la zona intertropical, tiene gran importancia dentro de la economía del Ecuador quien es exportador desde 1830 del cacao fino de aroma a base de la variedad conocida como Nacional (Amores, 1999). En la actualidad la superficie cultivada en el país de cacao se estima en aproximadamente 362,120 hectáreas, con una producción anual de 95,000 t y un rendimiento de 0.27 $\mathrm{t} \mathrm{ha}^{-1}$, lo que significa 5.40 quintales por hectárea al año (SICA, 2005).

El Banco de germoplasma de cacao variedad "Nacional" existente en la finca experimental "La Buseta" propiedad de la Universidad Técnica Estatal de Quevedo, es el resultado de la colección sistemática de muestras representativas de cacao Nacional existentes en la región desde la década del 40 por la United Fruit. Sin embargo, esta caracterización se ha basado en caracteres fenotípicos, directamente influenciados por factores ambientales, herencia multigénica, herencia cuantitativa y por la dominancia parcial de algunos caracteres, la cual puede confundir la expresión de un rasgo genético (Wilde et al., 1992).

El mejoramiento genético en cultivos perennes puede tomar varios años y en el caso del cacao puede llevar de 10 a 12 años hasta lograr un nuevo cultivar (Díaz, 2001). Este proceso puede ser acelerado mediante el uso de técnicas moleculares, las cuales han demostrado ser herramientas útiles para el mejoramiento genético y para la selección de variedades con características importantes (Araujo citado por Faleiro et al., 2001). Con la identificación de marcadores moleculares asociados a loci que codifican tanto para características cualitativas como cuantitativas (QTL's), por su acrónimo en ingles, es posible realizar selección asistida por marcadores.

La presente investigación busca identificar y caracterizar clones de cacao de la variedad Nacional de las cuales existen registros de comportamiento en campo, utilizando marcadores moleculares RAPD's, la cual es una de las herramientas más adecuadas para estudios genéticos, ya que superan en la mayoría de los casos las limitaciones de los métodos tradicionales (Williams et al., 1990), además se lograría una mejor reclasificación de los materiales en grupos de determinadas tendencias y sería de incalculable ayuda para trabajos a mediano plazo en la obtención de progenies con características estabilizadas gracias al descarte de plántulas inestables con las cuales se podría iniciar programas de mejoramiento genético. Dado lo anterior el objetivo de este estudio fue estimar la diversidad genética de una colección de Theobroma cacao (L.) variedad Nacional usando marcadores RAPD's.

\section{MATERIALES Y MÉTODOS}

Las muestras foliares de los 20 accesiones de cacao, con fines de analizarlas molecularmente empleando la técnica de RAPD's ("Polimorfismos del ADN Amplificado al Azar"), fueron recolectadas en la Finca experimental "La Buseta" identificadas, luego llevadas al laboratorio del programa de Biotecnología de la Universidad de Guayaquil para la extracción del ADN y las reacciones de AP-PCR (Cuadro 1).

El análisis estadístico se basó en la codificación de cada banda polimórfica que se representa a través de las variables presencia (1) o ausencia (0) los mismos que fueron procesados a través del método de agrupamiento UPGMA (Unweighted Paired Prouping Metho with Arithmetic Average) y el coeficiente de similitud de Nei y Li (1979). Se registraron por inspección en una matriz de datos binarios en el programa NTEDIT del paquete estadístico NTSYS pc ver. 2.0 (Applied Biostatistics Inc 1998). No se tomaron en cuenta polimorfismos que involucren datos dudosos. Para la extracción de ADN de cacao se probaron algunos protocolos aplicados a tejidos vegetales, con la finalidad de obtener ADN de buena calidad y aplicable a todas las muestras sometidas al proceso de extracción entre ellos: 2 Protocolos propuestos por el Programa de Biotecnología, así como otros propuestos por varios autores como (Uyemoto et al., 1998; Osorio et al., 2000; Ferreira y Grattapaglia, 1998; Colombo et al., 1998; Doyle y Doyle 1990).

El protocolo Doyle y Doyle (1990) modificado fue el que mejores resultados dio en cuanto a la calidad y concentración del ADN. La integridad y concentración del ADN fueron analizadas por electroforesis en minigeles de agarosa al $1.2 \%$ en tampón TAE $1 \mathrm{X}$, la cual se realizó a 90 voltios por $30 \mathrm{~min}$. (Ferreira y Grattapaglia, 1998).

Las muestras de ADN del material genético fueron amplificadas utilizando el protocolo propuesto por Faleiro (2000) (programado para 40 ciclos cada uno constituido por el primer segmento $4^{\prime \prime}$ a $94^{\circ} \mathrm{C}$, segundo segmento $15^{\prime \prime}$ a $94^{\circ} \mathrm{C}, 30^{\prime \prime}$ a $36^{\circ} \mathrm{C}, 90^{\prime \prime}$ a $72^{\circ} \mathrm{C}$, tercer segmento 7 minutos a $72{ }^{\circ} \mathrm{C}$, y Conservación a $4^{\circ} \mathrm{C}$ ). A cada muestra se colocó $3 \mu \mathrm{L}$ de una mezcla de azul de bromofenol (0.25\%) (Araujo, 1999). Para la mix de la PA-PCR en cada tubo de reacción se colocó una alícuota de los siguientes componentes: ADN (5 ng $\left.\mu \mathrm{L}^{-1}\right) 1.00$ $\mu \mathrm{L} 10 \mathrm{X}$ buffer, $5 \mu \mathrm{L} 50 \mathrm{mM}$ ClMg, $5 \mu \mathrm{L}$ cebador $(1.0$ $\mu \mathrm{M}), 2.00 \mu \mathrm{L}$ dNTPs (2.5 mM cada uno), $4.00 \mu \mathrm{L}$ Taq

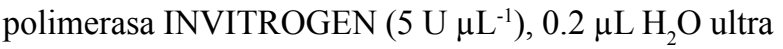
pura $31.8 \mu \mathrm{L}$ volumen final: $50.00 \mu \mathrm{L}$. Se probaron 14 
oligonucleótidos (OPA-02, OPA-04, OPA-07, OPA12, OPA-15, OPA-16, OPC-01, OPC-04, OPC-08, OPC-09, OPC-10, OPC-07, OPC-13, OPC-14), los mismos que han sido empleados en trabajos anteriores de caracterización y diversidad genética de cacao realizados por (Faleiro et al., 2002 y Araujo et al. 1999) respectivamente, en el Centro de Pesquisas do Cacau (CEPEC-CEPLAC) Ilhéus-Brasil.

Cuadro 1. Código y características de las 20 accesiones utilizados en el "Análisis de la diversidad genética de una muestra de la colección de Theobroma cacao (L.) existente en la Finca Experimental "La Buseta" 2007

\begin{tabular}{rlllll}
\hline & CODIGO & CARACTERÍSTICA & & CODIGO & \multicolumn{1}{c}{ CARACTERÍSTICA } \\
\hline $\mathbf{1}$ & L 11 H 19 & Productor & $\mathbf{1 1}$ & L 23 H 49 & Tolerante a Monilla \\
$\mathbf{2}$ & L 12 H 01 & Tolerante a Monilla & $\mathbf{1 2}$ & L 23 H 63 & Susceptible a Crinipellis \\
$\mathbf{3}$ & L 17 H 25 & Susceptible a Monilla & $\mathbf{1 3}$ & L 24 H 53 & Susceptible Ceratocistys \\
$\mathbf{4}$ & L 17 H 26 & Susceptible a Monilla & $\mathbf{1 4}$ & L 43 H 64 & Resistente a Monilla \\
$\mathbf{5}$ & L 18 H 58 & Productor, Resistente Monilla & $\mathbf{1 5}$ & L 26 H 64 & Productor \\
$\mathbf{6}$ & L 19 H 46 & Susceptible a Ceratocistys & $\mathbf{1 6}$ & L 30 H 50 & Resistente a Crinipellis \\
$\mathbf{7}$ & L 19 H 48 & Susceptible a Ceratocistys & $\mathbf{1 7}$ & L 30 H 62 & Susceptible a Crinipellis \\
$\mathbf{8}$ & L 22 H 40 & Resistente a Crinipellis & $\mathbf{1 8}$ & L 33 H 45 & Productor \\
$\mathbf{9}$ & L 30 H 06 & Susceptible a Ceratocistys & $\mathbf{1 9}$ & L 34 H 07 & Susceptible Ceratocistys \\
$\mathbf{1 0}$ & L 23 H 41 & Productor, Resistente Monilla & $\mathbf{2 0}$ & L 42 H 60 & Resistente a Crinipellis \\
\hline
\end{tabular}

\section{RESULTADOS Y DISCUSIÓN}

La extracción de $\mathrm{ADN}$ de cacao se la realizó a partir de hojas de cacao en estado intermedio de madurez, las cuales mostraron los mejores resultados, Con el protocolo de Doyle y Dolyle (1990) modificado, se pudo obtener ADN de buena calidad en mayor cantidad, sin degradación, que migró en el gel de agarosa como una banda única de alto peso molecular (Figura 1).

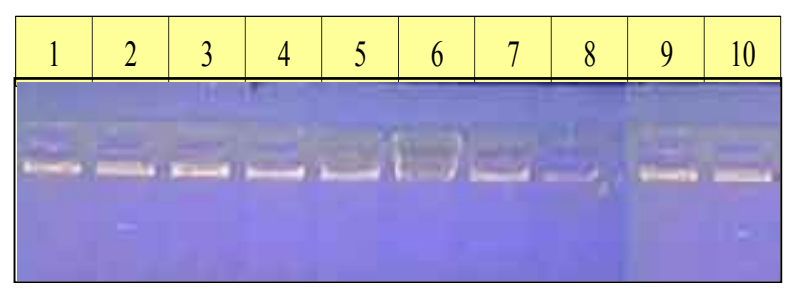

Figura 1. Resultados de la extracción de ADN genómico de 10 accesiones de Theobroma cacao (L.) en geles de agarosa teñidos con bromuro de etidio con el método de Doyle y Doyle 1990 modificado

Utilizando las condiciones óptimas de amplificación fue posible analizar la diversidad genética de 20 accesiones de cacao. De los nueve oligonuclótidos utilizados se generaron un total de 67 bandas con un buen nivel de repetibilidad. De ellas el 88\% (59) bandas mostraron perfiles polimórficos (Cuadro 2).
Se probaron 14 oligonucleótidos decámeros (Operon Technologies Inc., Alameda, CA, EUA) para la obtención de marcadores RAPD's con tres de las 20 accesiones, de los cuales 9 amplificaron bandas claras y reproducibles entre ellos OPA-15, OPC-07, OPC-9, OPC-4, OPC-3, OPC-1, OPA-12, OPC-13 у OPA-7 (Figura 2), estos fueron aplicados a las 20 accesiones con dos repeticiones mostrando perfiles polimórficos. (Figura 2).

\begin{tabular}{|c|c|c|c|}
\hline $\begin{array}{lr}\text { Cuadro 2. } & \mathbf{P} \\
& \mathbf{0} \\
& \mathbf{a} \\
& \mathbf{e} \\
\text { Código Operón }\end{array}$ & \multicolumn{3}{|c|}{$\begin{array}{l}\text { accesiones de Theobroma cacao (L.) existentes } \\
\text { en la finca la Buseta }\end{array}$} \\
\hline \multirow{2}{*}{ Código Operón } & \multicolumn{2}{|c|}{ Productos amplificados } & \multirow{2}{*}{$\begin{array}{c}\text { Polimorfismo } \\
(\%)\end{array}$} \\
\hline & Monomórficas & Polimórficas & \\
\hline OPA-15 & 1 & 8 & 87 \\
\hline OPA-03 & 2 & 9 & 77 \\
\hline OPC-04 & 2 & 3 & 33 \\
\hline OPC-13 & 1 & 5 & 80 \\
\hline OPA-07 & 0 & 6 & 100 \\
\hline OPC-09 & 1 & 7 & 85 \\
\hline OPA-12 & 0 & 8 & 100 \\
\hline OPC-01 & 1 & 7 & 85 \\
\hline OPC-07 & 0 & 6 & 100 \\
\hline TOTAL & 8 & 59 & \\
\hline
\end{tabular}




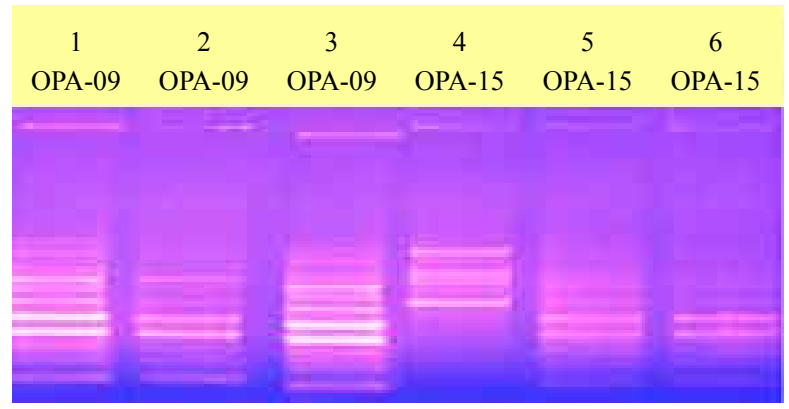

Figura 2. Productos de amplificación de DNA genómico de 3 accesiones de cacao usando los oligonucleótidos OPA 09 y OPA 15

Los datos obtenidos con los 9 cebadores permitieron la construcción de un dendrograma que mostró las relaciones genéticas entre las 20 accesiones de cacao (Figura 3). Este dendrograma mostró dos grandes grupos A y B, el grupo A se incluyó a las accesiones (L-30-H-62) que fenológicamente posee características de susceptibilidad a Crinipellis y la accesión (L-34H07) resistencia a Ceratocystis, y en el grupo B se encuentran las 18 accesiones restantes el cual incluye 2 subgrupos B1 y B2, en el subgrupo B1 se incluye la accesión (L-22-H40) el cual fenológicamente posee características de resistencia a Crinipellis. Y el subgrupo B2 en donde están incluidos las accesiones que poseen características de productividad.

En la amplificación al azar del ADN polimórfico (RAPD) para los 20 accesiones de Theobroma cacao(L.), los que produjeron los niveles más altos de diversidad fueron los primeros OPC 04 (0.80) y OPC 07 (0.82). El cebador que reportó el nivel más bajo fue OPC 01 (0.37). Las accesiones que presentaron los niveles de variabilidad más altos fueron (L-22H-40) (0.75), (L-34H07) (0.86) y (L-42-H60) (0.72) mientras que los valores más bajos los mostraron las accesiones (L-26-H64) (0.48) y (L-23-H63) (0.47). El valor de la diversidad total (HT) obtenida para los 20 accesiones de cacao (Theobroma cacao L.) mediante RAPD's fue de 0.636 .

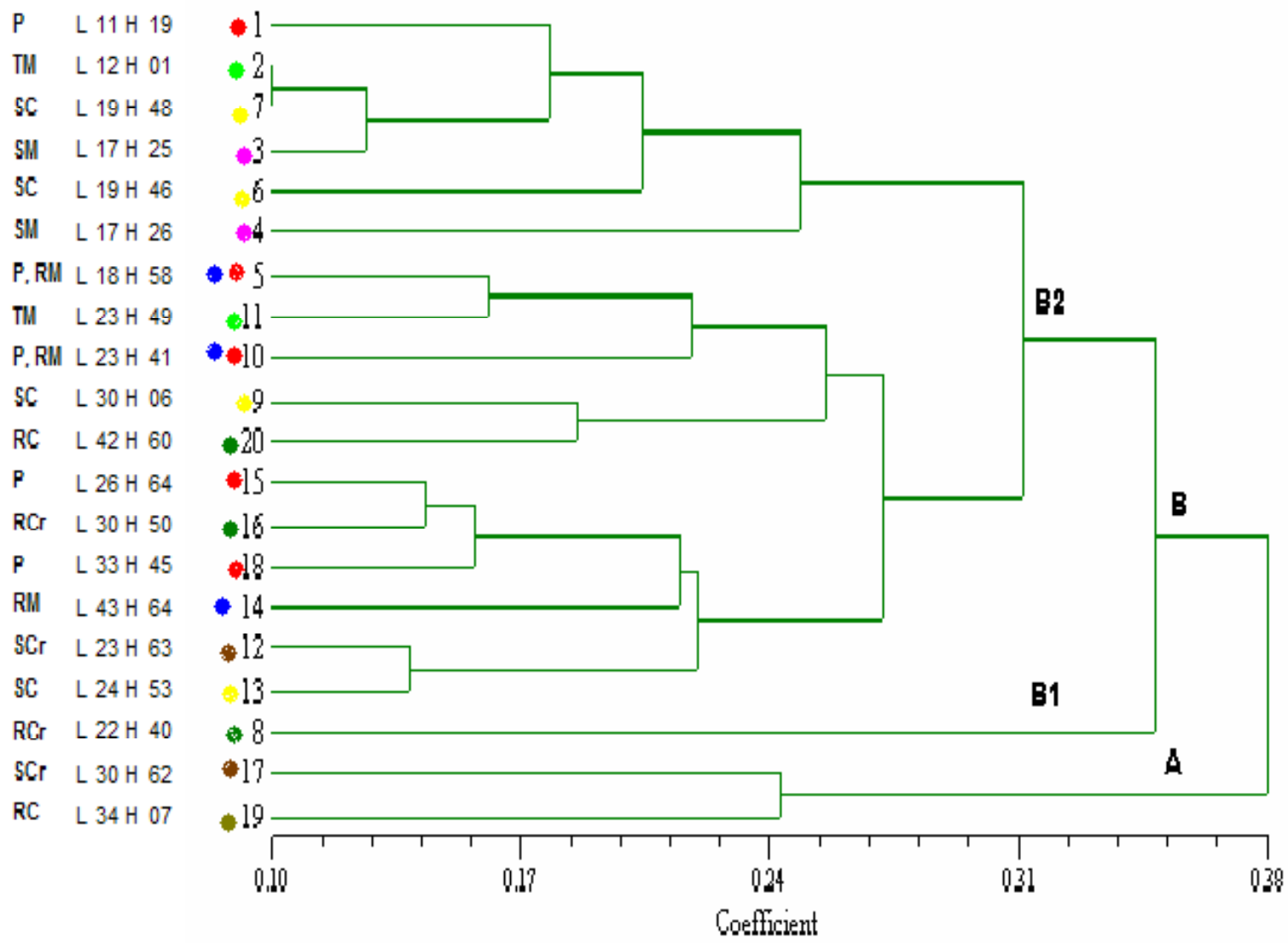

Figura 3. Dendrograma de las 20 accesiones de Theobroma cacao (L.) basado en los RAPDs obtenidos con el coeficiente de similitud de Nei y Li y el método de agrupamiento UPGMA (Los números indican a cada accesión. Las letras A y $B$ representan cada una de las ramas o grupos principales del árbol y las letras B1 y B2 correspondes al los sub grupos). RCr resistencia Crinipellis, $\mathbf{R C}$ Resistencia a Ceratocistys, $\mathbf{P}$ Productores, SC Susceptible a Ceratocistys, SCr Susceptible a Crinipellis, RM Resistencia a Monilla TM Tolerante a Monilla, SM Susceptible a Monilla 


\section{CONCLUSIONES}

En base a los resultados obtenidos se concluye que el mejor protocolo de extracción de ADN de Theobroma cacao (L.) fue el propuesto por Doyle y Doyle (1990) con ciertas modificaciones. Los oligonucleótidos OPC-4 y OPC-7 mostraron los niveles de diversidad más altos. Los niveles de diversidad media para los oligonucleótidos usados en todas las muestras fue de $(\mathrm{Ht}=0.63)$. Los porcentaje de bandas polimórficas para las 20 accesiones de cacao fue $88 \%$ con los nueve oligonucleótidos. Las accesiones con características de productividad mediante el análisis RAPD fueron agrupadas en el mismo grupo B2.

Con el propósito de contribuir con la selección asistida por marcadores (MAS) es imperioso desarrollar otros marcadores moleculares hasta alcanzar la cobertura completa del genoma de Theobroma cacao, como los AFLP (Amplificación de fragmentos de longitud polimorfica) y SSCP (Polymerase chain reaction singlestrand conformation polymorphism).

\section{LITERATURA CITADA}

Amores, F. 1999. La dificultad para establecer el rumbo tecnológico que han limitado el impacto económico de la investigación de cacao durante los últimos 50 años Quito - Ecuador, tesis de maestría. Universidad Internacional SEK pag. 188.

Araújo, L. G., Prabhu, A.S. \& Fillipi, M.C. 1999. Genetic analysis of leaf blast resistance in somaclones of rice cultivar Araguaia. Fitopatologia Brasileira 24:182-184

Doyle, J. J.; Doyle, J. L. 1990. Isolation of plant DNA from fresh tissue. Focus 12: 13-15.

Faleiro, F., Araújo, I., Bahia, R., Santos, R., Yomada, M. y Anhert, D. 2002. Otimização da extração e amplificação de DNA de Theobroma cacao L. visando obtenção de marcadores RAPD. Agrotrópica (Brasil) 14(2): 31-34.

Faleiro, F. 2001. Proyecto BIOMOL/CEPEC/CEPLAC El uso de Técnicas Moleculares para la búsqueda de variedades resistentes a enfermedades de escoba de bruja en cacao. Ilhéus, Brasil, CEPLAC.

Ferreira, M. y Grattapaglia, D. 1998. Introducción al uso de marcadores moleculares en el análisis genético. EMBRAPA. Brasil. p 100-1005.

Osorio, M. 2000. Caracterización molecular de genotipos de cacao (Theobroma cacao L) tipo criollo pertenecientes a la colección 95 de la estación experimental de Ocumare de la Costa estado Aragua, mediante el uso de RAPD. Tesis de grado. Postgrado de Agronomía. Facultad de Agronomía - UCV. Maracay. 105p.
SICA. 2005. SERVICIO DE INFORMACIÓN AGROPECUARIA del MINISTERIO DE AGRICULTURA Y GANADERÍA DEL ECUADOR. ECUADOR: Producción mundial de cacao. Fuente:1991-1995- Sistema Estadístico Agropec. Nacional -INEC-1996-1999 Direcciones Provinciales -MAG- DIA *Proyecto ECU ANECACAO-Elaboración:Proyecto SICA/MAGEcuador (www.sica.gov.ec) 14/septiembre/2005.

Uyemoto JK Zhang yp, Kirkpatrick BC. 1998 A smallscale procedure for extracting nucleic acids from woody plants infected with various phytopathogens for PCR assay. USDA-ARS and Department of Plant Pathology, University of California, Davis 95616, USA. 